Chapman University

Chapman University Digital Commons

Pharmacy Faculty Articles and Research

School of Pharmacy

7-1-2015

\title{
Effect of siRNA pre-Exposure on Subsequent Response to siRNA Therapy
}

Hamidreza Montazeri Aliabadi

Chapman University, montazer@chapman.edu

Parvin Mahdipoor

University of Alberta

Cezary Kucharski

University of Alberta

Nicole Chan

University of Alberta

Hasan Uludag

University of Alberta

Follow this and additional works at: http://digitalcommons.chapman.edu/pharmacy_articles

Part of the Cancer Biology Commons, Medical Biochemistry Commons, Medical Genetics Commons, and the Oncology Commons

\section{Recommended Citation}

Aliabadi, H.M., Mahdipoor, P., Kucharsky, C., Chan, N., Uludağ, H., 2015. Effect of siRNA pre-Exposure on Subsequent Response to siRNA Therapy. Pharm Res 1-14. doi:10.1007/s11095-015-1741-z

This Article is brought to you for free and open access by the School of Pharmacy at Chapman University Digital Commons. It has been accepted for inclusion in Pharmacy Faculty Articles and Research by an authorized administrator of Chapman University Digital Commons. For more information, please contact laughtin@chapman.edu. 


\section{Effect of siRNA pre-Exposure on Subsequent Response to siRNA Therapy}

\section{Comments}

This is a pre-copy-editing, author-produced PDF of an article accepted for publication in Pharmaceutical Research in July 2015 following peer review. The final publication is available at Springer via DOI: 10.1007/ s11095-015-1741-z

\section{Copyright}

Springer 


\title{
Effect of siRNA pre-exposure on subsequent response to siRNA therapy
}

\author{
Hamidreza Montazeri Aliabadi ${ }^{*}, \mathrm{a}, \mathrm{b}$, Parvin Mahdipoor $^{\mathrm{b}}$, Cezary Kucharsky ${ }^{\mathrm{b}}$, \\ Nicole Chan ${ }^{\mathrm{b}}$, Hasan Uludağ ${ }^{*}, \mathrm{~b}, \mathrm{c}, \mathrm{d}$
}

${ }^{a}$ School of Pharmacy, Chapman University, Irvine, CA 92618, USA

${ }^{\mathrm{b}}$ Department of Chemical \& Material Engineering, Faculty of Engineering, University of Alberta, Edmonton, AB, Canada, T6G 2G6

${ }^{c}$ Faculty of Pharmacy and Pharmaceutical Sciences, University of Alberta, Edmonton, AB, Canada, T6G 2E1

${ }^{\mathrm{d}}$ Department of Biomedical Engineering, Faculty of Medicine and Dentistry, University of Alberta, Edmonton, AB, Canada, T6G 2R3

\section{* Corresponding Authors}

Hasan Uludă̆, Ph.D.

\#2-020 RTF, Chemical \& Materials Engineering Department,

Faculty of Engineering, University of Alberta, Edmonton, Alberta, Canada T6G 2G6.

Tel: (780) 492-0344. Fax: (780) 492-2881. E-mail: huludag@ualberta.ca

Hamidreza Montazeri Aliabadi, PhD

School of Pharmacy Chapman University

\#211, 9401 Jeronimo Road

Irvine, CA 92618, USA

Tel: (714) 516-5492. Fax: (714) 516-5481. E-mail: montazer@ chapman.edu 


\begin{abstract}
PURPOSE. An alternative cancer therapy based on RNA interference (RNAi) has shown considerable promise but the possibility of resistance development is not known. This study explored the possibility of therapeutic resistance against siRNA nanoparticles in human cancer cells. METHODS. Two approaches to siRNA treatment were undertaken using lipid-modified polyethylenimines, a single high concentration (shock) and repeated increasing concentrations (gradual). The targets were Mcl-1, RPS6KA5 and KSP in MDA-MB-435 cells. RESULTS. There was no evidence of resistance development in shock-treated cells, while the decrease in mRNA levels of targeted proteins was not as robust in naïve cells in gradual treatment. However, silencing efficiency was restored after a 7-day recovery period when expression of suppressed proteins returned to normal levels. Cellular uptake of siRNA was not affected by pre-treatments. Other mediators involved in cell survival and proliferation were altered in siRNA-treated cells, but only JUN silencing led to a heightened loss of viability. In vivo experiments demonstrated similar silencing efficiency at mRNA level after repeat doses. CONCLUSIONS. Human cancer cells responded to repeat siRNA nanoparticles in a similar fashion after a temporary initial alteration and little, if any, resistance was evident against repeated siRNA treatments.
\end{abstract}

KEYWORDS: siRNA, cancer therapy, drug resistance, polymeric carriers,

\title{
LIST OF ABBREVIATIONS
}

FAM: Fluorescein amidite

KSP: kinesin spindle protein

Mcl-1: myeloid leukemia cell differentiation protein mRNA: messenger RNA

NT: Non-treated

PEI-LA: linoleic acid-substituted polyethylenimine

RISC: RNA-induced silencing complex

RQ: Relative quantity

RT-PCR: real-time polymerase chain reaction siRNA: short interfering RNA 


\section{INTRODUCTION}

Innate and acquired resistance against chemotherapeutic agents is an old problem and one of major causes of failure of cancer chemotherapy. While innate resistance is observed independent of preexposure to anticancer treatment and might be attributed to the phenotype of original malignant cells, acquired resistance is manifested after exposure to cytotoxic agents and could result in cross-resistance to other cytotoxic molecules with unrelated molecular structures ${ }^{1}$. Different mechanisms are involved in acquired resistance, which include, but are not limited to: (a) reduced intracellular uptake or enhanced efflux of drugs by membrane transporters such as P-glycoprotein and breast cancer resistance protein ${ }^{2,3}$; (b) inhibition of apoptosis by over-expression of apoptosis inhibitors such as Mcl-14, Bcl-2 $2^{5,6}$ and survivin $^{7}$; and (c) mutations in drug target genes, which are critical for resistance against latest generation of molecularly designed drugs, such as mutations in MEK (resistance against BRAF inhibitors) ${ }^{8}, E G F R$ (resistance against small molecule tyrosine kinase inhibitors) ${ }^{9}, B C R-A B L$ (resistance against dasatinib) ${ }^{10}$ and secondary mutations in RAS proteins NRAS or KRAS (resistance against vemurafenib and dabrafenib $)^{11}$. Once the resistance sets in, it becomes necessary to employ new drugs and/or sensitizing agents to make the malignant cells responsive to chemotherapy, which is usually not successful on the long run.

As an alternative to drug therapy, small interfering RNA (siRNA) mediated RNA interference (RNAi) are being intensely explored both as an investigational tool and as a therapeutic strategy ${ }^{12}$. In this approach, expression of a target protein is "silenced" at post-transcriptional stage by intracellular delivery of a double stranded RNA, where it is incorporated in the RNA-induced silencing complex (RISC), which contains the Argonaute 2 enzyme that is capable of "slicing" the mRNA target. After removal of the "passenger" strand, the remaining "guide" strand of siRNA will bind to complementary region of the target mRNA, which triggers cleavage and subsequent degradation of the mRNA ${ }^{13}$. The siRNA on its own is not effective due to its rapid degradation in physiological milieu and its inability to cross cell membranes; a carrier is employed to bind and protect it from degradation and form complexes to enable 
cellular uptake. It has been recognized that a large variation exists in the required siRNA copy number for effective silencing in different cell lines with different carriers ${ }^{14}$. Based on the estimated number of siRNA molecules delivered by a nanoparticle (e.g., 2000 copies in a $70 \mathrm{~nm}$ particle) ${ }^{15}$, it appears that only a small portion of the siRNA molecules exposed to cells is available for efficient silencing. More importantly, multiple exposures are usually required to accomplish a significant effect due to transient nature of siRNA mediated silencing. We have little information on the effect of siRNA pre-exposure on cellular response to subsequent siRNA treatments. While siRNA has the potential to curb tumor growth independent of chemotherapy, it is not known if it is susceptible to resistance development similar to conventional drugs. Should we expect appearance of cell populations that do not respond to siRNA silencing due to previous exposure? Can internalization of siRNA complexes be minimized as a result of siRNA pre-exposure, reminiscent of induction of efflux proteins (e.g., ABC membrane proteins) that minimize cellular accumulation of cytotoxic drugs? In the only study reported on the possibility of siRNA resistance, cellular adaptation was found to contribute to resistance to viruses; siRNA efficacy was inhibited by adenosine deaminases acting on RNA, which 'edits' the delivered siRNA by converting adenosine-to-inosine (A-to-I ${ }^{16}$. Considering these unresolved issues, a more in depth investigation of cellular responses to repeat siRNA treatments is warranted.

This study was designed to investigate the effect of siRNA pre-exposure on subsequent response of a human cancer model (MDA-MB-435 cells) to siRNA therapy. A systematic approach was undertaken to investigate this issue by using two siRNA exposure methods. The pre-exposure to siRNA was performed either by a single, high concentration of siRNA treatment or by multiple treatments with gradually increasing siRNA concentrations, which will be referred to as "shock" and "gradual" treatments, respectively. We selected three siRNAs that were previously shown to be effective in retarding growth in the chosen cell model: (i) an siRNA targeting myeloid cell leukemia 1 (Mcl-1) protein, (ii) an siRNA targeting kinesin spindle protein (KSP), and; (iii) a combination of siRNAs targeting Mcl-1 and RPS6KA5 proteins, whose efficacy was identified from a library screen of siRNA 
combinations $^{17}$. The effect of siRNA pre-exposure on MDA-435 cells was evaluated by investigating cellular internalization of siRNA, expression levels of targeted proteins at the mRNA level, and the resultant cellular viabilities. The cells were monitored during the siRNA treatments, as well as after a given period to analyze the cellular recovery after the siRNA therapy. An animal model was subsequently employed to confirm our findings in vivo.

\section{MATERIAL AND METHODS}

Materials. The 2 kilo Dalton (kDa) PEI (PEI2; Mn: 1.8 kDa, Mw: $2 \mathrm{kDa}$ ), anhydrous dimethylsulfoxide (DMSO), linoleyl chloride (C18:2 9Z,12Z; 99\%), Hanks’ Balanced Salt Solution (HBSS; with phenol red), trypsin/EDTA, and 3-(4,5-dimethyl-2-thiazolyl)-2,5-diphenyl-2H-tetrazolium bromide (MTT) were purchased from SIGMA (St. Louis, MO). Clear HBSS (phenol red-free) was obtained from Lonza Inc. (Allendale, NJ). RPMI 1640 medium, penicillin (10000 U/mL) and streptomycin (10 mg/mL), Taq DNA polymerase, M-MLV reverse transcriptase, and RNaseOUT ribonuclease inhibitor were provided by Invitrogen (part of Life Technologies, Grand Island, NY). Fetal bovine serum (FBS) was from PAA Laboratories (Etobicoke, Ontario). RDD buffer were purchased from Qiagen (Mississauga, ON). Trizol ${ }^{\circledR}$ reagent was provided by Ambion (Burlington, Ontario).

Cell Line. MDA-MB-435 (MDA-435) cells were a generous gift from Dr. Robert Clarke (Georgetown University, Washington, DC). The cells were cultured in RPMI 1640 medium supplemented with 10\% FBS, $100 \mathrm{U} / \mathrm{mL}$ penicillin, and $100 \mu \mathrm{g} / \mathrm{mL}$ streptomycin at $37^{\circ} \mathrm{C}$ and $5 \% \mathrm{CO} 2$. Cell cultures were considered confluent when a cell monolayer covered more than $80 \%$ of the flask surface (equivalent to $600,000-900,000$ cells $/ \mathrm{mL}$; approximately equivalent to 100,000 cells $/ \mathrm{cm}^{2}$ ). To propagate the cells, the monolayer was washed with HBSS and treated with $0.05 \%$ Trypsin/EDTA at room temperature. The 
suspended cells were centrifuged at $600 \mathrm{rpm}$ for 5 minutes, re-suspended in fresh medium, and subcultured at $10 \%$ of the original count.

siRNAs and Primers. The scrambled siRNA (catalogue no: AM4635; used as negative control), Fluorescein amidite (FAM)-labeled scrambled siRNA (catalogue no: AM4620; used in uptake studies), and the siRNAs targeting KSP (catalogue no: AM16704; Ambion Silencer ${ }^{\circledR}$, unmodified) and ribosomal protein S6 kinase, polypeptide 5 (RPS6KA5; Catalogue number: AM51334; Ambion Silencer ${ }^{\circledR}$, unmodified) were provided by Ambion (Burlington, Ontario). The siRNA against induced myeloid leukemia cell differentiation protein (Mcl-1; catalogue no: SI02781205; Flexitube ${ }^{\circledR}$ siRNA) was supplied by Qiagen (Mississauga, ON). The primers used for the RT-PCR reactions were designed using the Primer-Blast software available at The National Center for Biotechnology Information website (http://www.ncbi.nlm.nih.gov/) and were synthesized with the following sequences by the IDT Technologies (Coralville, Iowa), before being validated in house:

$\beta$-actin (as endogenous protein): $\quad$ Forward: 5'-CCA CCC CAC TTC TCT CTA AGG A-3'

Reverse: 5'-AAT TTA CAC GAA AGC AAT GCT-3'

eIF4:

Forward: 5'-GAG GAC GAT GGC TAA TTA CAT TGA-3'

Reverse: 5'-GCA CAG AAG TGT CTC TAG CCA AAA-3'

FOS:

Forward: 5'-CTG AAG ACC GAG CCC TTT GA-3'

Reverse: 5'-GGA GCG GGC TGT CTC AGA-3'

JAK2:

Forward: 5'-AAC TGC AGA TGC ACA TCA TTA CCT-3'

Reverse: 5'-TCG AAA TTG GGC CAT GAC A-3'

JUN:

Forward: 5'-GAG AGG AAG CGC ATG AGG AA-3'

Reverse: 5'-TCC AGC CGG GCG ATT-3'

KSP:

Forward: 5'-TCA CAA AAG CAA TGT GGA AAC CTA-3'

Reverse: 5'-TCT GTC CAA AGA TTCA TTA ACT TGC A-3'

Mcl-1:

Forward: 5'-CCT TTG TGG CTA AAC ACT TGA AG-3'

MYC:

Reverse: 5'-CGA GAA CGT CTG TGA TAC TTT CTG-3'

Forward: 5'-TGG TCT TCC CCT ACC CTC TCA-3'

Reverse: 5'-AGA ATC CGA GGA CGG AGA GAA-3'

NFkB:

Forward: 5'-AAT GGG CTA CAC CGA AGC AA-3'

Reverse: 5'-CAG CGA GTG GGC CTG AGA-3'

RPS6KA5:

Forward: 5'-GAC ACT GCA GCC CAG CAA-3'

Reverse: 5'-CCT AAG CTA CTG AGT CCG AGA ACT G-3' 
S6K:

STAT3:

Survivin:
Forward: 5'-AAT CCG ATC ACC TCG AAG ATT TAT-3'

Reverse: 5'-CTG TGC TGG CCG AAG CA-3'

Forward: 5'-ACA ACA TGT CAT TTG CTG AAA TCA-3'

Reverse: 5'-TCC TTG GGA ATG TCA GGA TAG AG-3'

Forward: 5'-CCC CTC GGG CCA ACT G-3'

Reverse: 5'-CAG TTT GGC TTG CTG GTC TCT-3'

Preparation of siRNA Complexes. Lipid modification method to functionalize $2 \mathrm{kDa}$ PEI for siRNA delivery was previously described ${ }^{18,19}$. For this study siRNA complexes were formed by mixing selected siRNA(s) with a linoleic acid-substituted PEI (PEI-LA; 2.1 LA/PEI) at a polymer:siRNA weight/weight ratio of 8:1. Detailed complex formation procedure and characterization of these complexes, as well as silencing model (GFP) and endogenous proteins, have been reported previously ${ }^{20}$.

Pre-exposure to siRNA. The cells underwent siRNA treatment by using two distinct approaches: (1) Shock method: The MDA-435 cells were seeded in 6-well plates at 20\% confluency. After 24 hours incubation at $37^{\circ} \mathrm{C}$, cells were treated with a relatively high dose of siRNA with the final concentration of $54 \mathrm{nM}$ in culture medium. Surviving cells were collected by trpsinization after 72 hours of siRNA exposure and were transferred to a cell culture flask to recover or for specific experiments. (2) Gradual method: Cells were seeded under the same conditions as the "shock" method; however, the exposure to siRNA was performed with gradually increasing concentrations of 9,18 , and $27 \mathrm{nM}$, with a final concentration of $27 \mathrm{nM}$ as a repeat treatment. The cells were collected after $72 \mathrm{~h}$ exposure to each concentration and seeded in a new plate (at $20 \%$ confluency) for the next treatment. After the second $27 \mathrm{nM}$ exposure, surviving cells were collected and transferred to a cell culture flask to recover. The preexposure procedures are summarized in Supplementary Figure 1. 
Cellular Internalization of siRNA. The effect of pre-exposure to siRNA on subsequent cellular internalization was evaluated in naïve cells and cells exposed to siRNA by "shock" and "gradual" methods. The desired cells were seeded in 24 -well plates at $\sim 50 \%$ confluency, and incubated at $37{ }^{\circ} \mathrm{C}$ for $24 \mathrm{~h}$. Cells were then exposed to the complexes prepared with PEI-LA/FAM-labeled scrambled siRNA or non-labeled scrambled siRNA (as negative control) with a polymer:siRNA ratio of 8:1 and final siRNA concentration of $18 \mathrm{nM}$. After $24 \mathrm{~h}$ incubation, cells were washed with clear HBSS and suspended in clear trypsin before being fixed in a $3.7 \%$ formaldehyde solution. A Beckman Coulter QUANTA SC flow cytometer was used to quantify the average uptake and the siRNA-positive population. The FL1 channel of the flow cytometer was used to quantify the cell-associated FAM fluorescence. A "No Treatment (NT)" group was added to each set of experiment to gate the analysis and ensure that the auto-fluorescent cell population represented only 1-2\% of the total cell population.

Laser Scanning Confocal Microscope was also used for visual inspection of internalized siRNA complexes. Naïve and siRNA pre-exposed cells were seeded in 6-well plates containing glass slides in each well. After exposure to complexes formed with PEI-LA and FAM-labeled or non-labeled siRNA (30 nM siRNA concentration) for 24 or $72 \mathrm{~h}$, cells were washed with clear HBSS and fixed in a $3.7 \%$ formaldehyde solution for 30 minutes in room temperature. The fixed cells were then washed with HBSS before being stained with a $1 \mu \mathrm{g} / \mathrm{mL}$ solution of tetramethylrhodamine wheat germ agglutinin (WGA) conjugate that selectively binds to $\mathrm{N}$-acetylglucosamine and $\mathrm{N}$-acetylneuraminic acid residues in cell membrane ${ }^{21}$. After 2 minutes incubation with the dye at $37^{\circ} \mathrm{C}$, cells were washed with HBSS twice, and the glass slide was mounted with a medium (polyvinyl alcohol in glycerol) containing $1 \mu \mathrm{gg} / \mathrm{mL}$ 4',6diamidino-2-phenylindole (DAPI; Life Technologies, Grand Island, NY). Prepared slides were studied with a Zeiss LSM710 LSCM. 
Real-time Polymerase Chain Reaction (RT-PCR). The efficiency of siRNA silencing in naïve and siRNA pre-exposed cells was investigated at the mRNA level using RT-PCR. Cellular RNA was extracted by the Trizol ${ }^{\circledR}$ protocol. Briefly, cells were lysed with Trizol $\left(1 \mathrm{~mL}\right.$ for each $1 \times 10^{6}$ cells $)$ and incubated at room temperature for 5 minutes. Chloroform $(0.2 \mathrm{~mL}$ for each $\mathrm{mL}$ of Trizol $)$ was added to lysed samples, and after vigorous shaking and 2-3 minutes incubation at room temperature, the aqueous phase was separated. RNA was then precipitated with isopropanol and the pellet was washed with $75 \%$ ethanol. Extracted RNA was dissolved in RNAse free water, and the total RNA was determined by Nanodrop Lite (Thermo Fisher Scientific, Wilmington, DE). RNA $(0.5 \mu \mathrm{g})$ was reverse transcribed to synthesize cDNA by using random hexamer primer and dNTP mix at $65^{\circ} \mathrm{C}$ for 5 min. Synthesis buffer $(5 \times)$, DTT $(0.1 \mathrm{M})$, and RNAout RNase inhibitor $(1.8 \mathrm{U} / \mu \mathrm{L})$ were then added and the solution was incubated at $37{ }^{\circ} \mathrm{C}$ for $2 \mathrm{~min}$. MMLV RT enzyme was added and samples were incubated at $25{ }^{\circ} \mathrm{C}$ for $10 \mathrm{~min}, 37^{\circ} \mathrm{C}$ for $50 \mathrm{~min}$, and $70^{\circ} \mathrm{C}$ for $15 \mathrm{~min}$. An ABI $7500 \mathrm{HT}$ instrument (Applied Biosystems, now part of Life Technologies, Grand Island, NY) was used for RT-PCR analysis using human $\beta$-actin as the endogenous gene. All the primers were tested to assure equal efficiency (with a slope $<0.1$ for the $\Delta \mathrm{CT}$ vs. cDNA dilution graph for different primers designed for each protein of interest), and a template concentration of $10 \mathrm{ng} / \mu \mathrm{L}$ was determined as the optimal concentration based on the standard curves. Analysis was performed by calculating $\Delta \mathrm{C}_{\mathrm{T}}, \Delta \Delta \mathrm{C}_{\mathrm{T}}$, and relative quantity (RQ) using endogenous gene and "no treatment" group as reference points.

Evaluation of Cell Viability after siRNA Treatment. The effect of siRNA silencing on the number of viable cells was evaluated during and after each exposure by a standard MTT assay as reported previously ${ }^{20}$. The cells were exposed to indicated siRNA complexes for $72 \mathrm{~h}$ before the assay, and the MTT assay was performed during the stages of gradual treatment method, after completion of both exposure methods (using different siRNA concentrations selected based on the efficacy of each treatment). 
Animal Studies. All experiments were performed according to the University of Alberta guidelines for the care and use of laboratory animals. 4-6 weeks old female NCR nu/nu nude mice (Taconic Farms) were kept in a bio containment unit for at least one week and then $\sim 2$ million MDA435WT were subcutaneously injected into the right flank of the mice. Tumor growth was monitored every 72-96 hours by volume measurements that were performed by a digital caliper. Animals were included in the study when developed a tumor of $50-100 \mathrm{~mm}^{3}$ (length $\times$ width $^{2} \times 0.4$ ). Tumor-bearing animals were randomly assigned to two different treatment groups: animals treated with scrambled siRNA (CsiRNA) and animals treated with Mcl-1/RPS6KA5. Complexes were prepared with a PEI-LA2.1:siRNA w/w ratio of 8:1 and each animal received $1.5 \mu \mathrm{g}$ of each siRNA/mouse $(\sim 0.06 \mathrm{mg} / \mathrm{kg} /$ day; $3 \mu \mathrm{g}$ or $0.12 \mathrm{mg} / \mathrm{kg} /$ day total for CsiRNA group) via subcutaneous injections by the tumor site. Injections were administered at 7-days intervals (to allow recovery from the silencing effect of injections) for a total of three injections. A total of 18 mice were assigned to each group, and they were euthanized as followed:

3 mice: 72 hours after $1^{\text {st }}$ injection (to analyze the silencing efficiency by PCR)

3 mice: 7 days after $1^{\text {st }}$ injection (to evaluate recovery of targets and as baseline for second injection)

3 mice: 72 hours after $2^{\text {nd }}$ injection (to analyze the efficiency of repeated silencing by PCR)

3 mice: 7 days after $2^{\text {nd }}$ injection (to evaluate recovery of targets and as baseline for third injection)

3 mice: 72 hours after $3^{\text {rd }}$ injection (to analyze the efficiency of repeated silencing by PCR)

At the end of treatment period, tumors were extracted and stored in RNAlater ${ }^{\circledR}$ in $-20^{\circ} \mathrm{C}$. Tumor samples were homogenized for RNA extraction and q-PCR analysis.

\section{Statistical Analysis}

The significance of the changes in siRNA cellular internalization (calculated both as mean fluorescence and percentage of cells positive for labeled siRNA), mRNA levels (analyzed by RT-PCR), and cell viability were evaluated using Student's $t$-test $(\mathrm{p}<0.05)$. Standard deviations were calculated for all results shown and are represented as the error bar in all figures. 


\section{RESULTS}

\section{Shock siRNA Treatment}

In order to confirm the initial responsiveness of MDA-435 cells to selected siRNAs, cells were exposed to $54 \mathrm{nM}$ of scrambled, Mcl-1, Mcl-1/RPS6KA5, and KSP siRNAs for 72 hours, and obtained cell viabilities are summarized in Figure 1a. While the negative control (scrambled siRNA) showed a more significant response than expected at the chosen concentration $(54 \mathrm{nM})$, a greater decrease in cell viability was observed for all selected siRNAs, with a significant additive effect for the combination of Mcl-1 and RPS6KA5 siRNAs as reported before ${ }^{17}$. After the recovery ( 7 days after the treatment), the cells were treated with selected siRNAs again using a range of concentrations $(9-54 \mathrm{nM})$. The response of the pre-treated and the naïve cells to scrambled siRNA, as well as Mcl-1, and KSP siRNAs showed a similar pattern (Figure 1b), with a slight decrease in the level of response to $18 \mathrm{nM}$ siRNA targeting KSP in the pre-treated cells compared to naïve cells, which disappeared at the higher dose of $27 \mathrm{nM}$. The decrease in the viability with Mcl-1/RPS6KA5 siRNA combination was significantly lower in cells previously exposed to the same combination at $54 \mathrm{nM}$ compared to naïve cells $(90.8 \pm 5.5 \%$ vs. $73.9 \pm$ $12.0 \%$ for $18 \mathrm{nM}$ siRNA and $64.7 \pm 4.5 \%$ vs. $28.1 \pm 6.7 \%$ for $27 \mathrm{nM}$ siRNA, respectively).

In order to evaluate changes in the expression level of selected proteins, RT-PCR analysis was performed after 24 and 72 hours of shock siRNA treatment. The 24-hour time point represented the initial response to siRNA exposure while the 72-hour time point represented mRNA levels in surviving cells. After 24 hours, significant decreases in mRNA level of targeted proteins $(\sim 86 \%$ decrease in Mcl-1 mRNA for both Mcl-1 and Mcl-1/RPS6KA5 treated cells, 86\% decrease for RPS6KA5 mRNA for combinational treated cells, and $\sim 93 \%$ decrease in KSP mRNA in cells treated with KSP siRNA) were observed in all treatment groups (Figure 1c). It is noteworthy that the scrambled siRNA also showed a significant decrease in mRNA level of all monitored proteins, which was most significant for KSP ( 50\% 
of the "no treatment" expression level). However, there was a greater decrease in the mRNA levels for cells treated with specific siRNAs. The expression levels of all selected proteins were significantly higher after 72 hours (vs. 24 hours) in cells exposed to scrambled siRNA. In fact, this expression levels were even higher than the naïve cells, which was more significant for Mcl-1 and KSP. In specific siRNA treated cells, the mRNA levels were also higher than the levels at the 24-hour time point, but not as high as the mRNA levels in naïve cells. The RT-PCR analysis was repeated after 7 and 14 days for naïve and treated cells to explore changes in mRNA levels after recovery. After 7 days (Figure 1d), mRNA levels for the selected proteins showed a significant increase over the initial response. However, the mRNA levels of specifically targeted proteins were still lower than the naïve cells. After 14 days (Figure 1e), the mRNA levels of targeted proteins returned to initial levels (i.e., equivalent to naïve cells). Finally, the repeat siRNA silencing (with $54 \mathrm{nM}$ siRNA) after 7 days of initial treatment showed a similar responsiveness in naïve and shock-treated cells (Figure 1f). However, the effect of scrambled siRNA was not always similar in naïve and recovered cells: while the mRNA level of Mcl-1 showed a more significant decrease in recovered, scrambled siRNA treated cells, KSP mRNA levels was significantly lower in naïve cells.

Cellular internalization of FAM-siRNA particles was analyzed using confocal microscopy at two time points ( 24 and 72 hours after treatment), as well as flow cytometry after 24 hours exposure. The mean fluorescence and percentage of FAM-siRNA positive cells were negligible for particles formed with non-labeled siRNA, as expected. Significant uptake was evident for cells exposed to FAM-siRNA complexes in naïve and shock treated cells (Figures $\mathbf{2} \mathbf{a}$ and $\mathbf{2 b}$, respectively). The siRNA uptake in cells previously exposed to Mcl-1/RPS6KA5 and KSP siRNAs was significantly lower than the naïve cells. Confocal microscopy analysis after 24 hours revealed a similar uptake pattern that showed the complexes inside the cytoplasm, but a non-homogenous uptake pattern among the cells, with some cells showing $>10$ particles internalized, while other cells seemed particle-free (Figure 2c). At 72 hours after treatment, 
most internalized particles disappeared, with few particles remaining in all cells and no obvious differences among the study groups.

\section{Gradual siRNA Treatment}

The cells were monitored for their responsiveness to gradually increasing siRNA after each treatment. Figure 3a summarizes the response of the naïve cells as well as cells treated with specific siRNAs (exposure to 18 and $27 \mathrm{nM}$ siRNA after exposure to 9 and $18 \mathrm{nM}$ siRNA concentrations, respectively), where cell viability was compared to "No Treatment" group. Pre-exposure to a lower concentration of siRNA did not seem to affect the subsequent cell response, where similar pattern of cell viability was observed as the result of exposure to higher siRNA concentration in the next treatment. A similar experiment (with a wider range of siRNA concentrations) was performed 7 days after the completion of gradual exposures (Figure 3b). For cells exposed to scrambled or Mcl-1 siRNA, the response was similar to naïve cells, with the exception of the response at $54 \mathrm{nM}$ scrambled siRNA (where cells pre-exposed to scrambled siRNA showed a lower response compared to naïve cells) and $27 \mathrm{nM}$ Mcl-1 siRNA (where a similar difference was observed). For Mcl-1/RP6KA5 siRNA combination or KSP siRNA, pre-exposed cells showed lower response compared to naïve cells, especially at higher concentrations of siRNA.

The silencing efficiency was also evaluated at the mRNA level after 24 and 72 hours of each exposure (Figure 4). For the first exposures ( 9 nM siRNA) representing the initial response of naïve cells, the decrease in mRNA level was minimal after 24 hours, where a significant difference was observed only for the Mcl-1/RPS6KA5 and KSP treatment groups. This difference disappeared after 72 hours, and in fact, a higher level of mRNA was observed in both scrambled and KSP siRNA treated cells. For the second exposures (18 nM siRNA), a significant drop in mRNA levels for all targeted proteins was observed (but not with scrambled siRNA) after 24 hours of exposure; however, the level of response was significantly lower in cells pre-exposed to Mcl-1 and RPS6KA5 siRNAs. This difference was not 
significant for KSP treatment. A similar trend was observed after 72 hours for cells co-treated with Mcl1 and RPS6KA5 siRNAs. The 72-hour KSP mRNA level in cells pre-exposed to $9 \mathrm{nM}$ of KSP siRNA was significantly higher than the corresponding group at the earlier time point. A similar trend was not observed after the third stage of exposure ( $27 \mathrm{nM}$ siRNA), but re-appeared after the second exposure to $27 \mathrm{nM}$ siRNA (the last stage of gradually increasing siRNA exposures). On the other hand, after a significant improvement in silencing with increasing siRNA concentration from 9 to $18 \mathrm{nM}$, the second increase in siRNA concentration (from 18 to $27 \mathrm{nM}$ ) did not yield a similar increase in silencing in naïve or pre-treated cells.

The RT-PCR analysis was repeated after 7 and 14 days of the last siRNA treatment (Figure 5). The recovery process was slower for the gradual siRNA treatment, where the levels of targeted mRNAs after 7 days (except KSP) was lower than the corresponding time point in the shock treatment (Figure 5a; compare to Figure 1d). This difference, however, disappeared after 14 days (Figure 5b), where the mRNA levels returned to similar levels as the naïve cells. Similar to the shock-treated cells, a repeat silencing with high siRNA concentration $(54 \mathrm{nM})$ after 7 days of completion of gradual exposures showed no significant difference in mRNA levels of naïve and pre-treated cells (Figure 5c).

The flow cytometry results for siRNA uptake in naïve and cells treated with gradual siRNA exposures are summarized in Figures $\mathbf{6 a}$ and $\mathbf{6 b}$; no negative impact on cellular uptake of polymer/siRNA particles was observed as a result of previous siRNA treatment. In fact, the mean fluorescence in cells pre-exposed to gradual siRNA against KSP was even higher than the naïve cells (Figure 6b). No significant difference was observed in the percentage of FAM-siRNA positive cells for pre-treated cells compared to the naïve cells. The confocal microscopy evaluations revealed a similar cellular internalization to our observations with cells pre-treated with the "shock" method; while significant (but non-homogeneous) uptake was apparent after 24 hours, particles seemed to dissociate after 72 hours, where few fluorescent particles was observed in selected cell populations. 


\section{Targeting Related Proteins in siRNA-Treated Cells}

To investigate the effect of siRNA treatments on expression of other proteins potentially involved in cell survival, mRNA levels of a select group of proteins were analyzed 24 and 72 hours after the shock treatment and the last step in gradual exposures to Mcl-1 and Mcl-1/RPS6KA5 siRNAs. The initial (24 hours) response to shock treatment was a reduction of the targeted proteins (as expected), as well as a reduction in mRNA levels of survivin (both treatment groups), JAK2, eIF4, and NFKB (more significant in Mcl-1/RPS6KA5 combination group), MYC, S6K, and STAT3 (only in Mcl-1/RPS6KA5 combination group) (Figure 7a). For cells gradually exposed to siRNA, on the other hand, a reduction could only be observed in survivin mRNA, while several proteins (JUK, NFkB and MYC in particular) showed an overexpression as a result of multiple exposures over time (Figure 7b). At 72 hours, a reduction in mRNA levels of targeted proteins was seen, while a significant overexpression was observed in STAT3 and JUN in both pre-exposed cell populations (Figure $\mathbf{7 b}$ and $\mathbf{7 d}$ ). A significant increase in NFkB expression was only observed in the gradual treatment (Figure 7d).

We next treated the shock- and gradual-treated cells by specific siRNAs against significantly altered mediators, namely JAK2, STAT3, JUN, NFkB, MYC or S6K, and the viability of the cells was compared to untreated MDA435 cells (Figure 7e). The MYC and S6K were selected as negative controls since they did not show a significant increase with siRNA-treated cells. While the responses to most siRNAs were not altered in the pre-treated cells, JUN-specific siRNA showed a significant effect on the viability of the cells pre-treated with Mcl-1 and Mcl-1/RPS6KA5 combination (emphasized by red

arrows). This was the case for both shock and gradual strategies, and this effect was more significant in cells pre-treated with Mcl-1/RPS6KA5 siRNA combination. The viability of the cells included in this experiment showed a similar trend as a response to silencing of other different proteins.

\section{siRNA Response in a Xenograft Model}


A xenograft model (human MDA-435 cells in nude mice) was employed to further investigate two aspects of our in vitro observations; the efficacy and changes in target protein expression with repeat siRNA injections. We previously reported the efficacy of siRNA therapies in a similar animal model with intratumoral injections ${ }^{17}$, but this time we employed a subcutaneous route for siRNA injections in order not to disrupt the tumor growth directly. Figure 8a summarizes the tumor growth pattern of treatment (Mcl-1/RPS6KA5 combination, as the most efficacious therapy in vitro) and negative control groups. Three subcutaneous injections were performed 7 days apart to allow enough time for recovery. While tumors in the control group showed a continuous growth, the treatment group showed a halting of tumor growth after each injection and a slight increase in tumor size until the repeat injection, leading no significant growth during the study period. RT-PCR analysis of mRNA levels of targeted proteins, as well as JUN (i.e., one protein overexpressed in pre-treated cells) is summarized in Figure $8 \mathbf{b}$. The mRNA levels was decreased by $40 \%$ (range: 34.6-42.3\%) for Mcl-1 and 45\% (range: 43.2-48.8\%) for RPS6KA5 72 hours after each injection, returning to previous levels as the control group after the recovery period. The JUN expression in Mcl-1/RPS6KA5 siRNA combination treatment was higher than the control group at all time points, despite some fluctuations during repeat siRNA treatments.

\section{DISCUSSION}

Although siRNA delivery has emerged as a promising strategy in cancer therapy, many details about the silencing process and factors affecting its efficiency remain unknown. The present study was designed to specifically investigate the effect of siRNA pre-exposure on subsequent response to siRNA treatments. Three siRNAs employed for this study have promising roles in anti-cancer therapy; (i) Mcl1 is among the anti-apoptotic proteins overexpressed in different cancers ${ }^{22}$, and promotes survival by inhibiting mitochondrial cytochrome c release ${ }^{23}$, (ii) Ribosomal protein S6 kinase family has been linked to critical proteins involved in cell survival, including NFkB, MYC, eIF4B, and c-FOS ${ }^{24}$ and RPS6KA5, in combination with Mcl-1, showed a significant therapeutic effect in retarding MDA-435 tumors in an 
animal model ${ }^{17}$, and; (iii) KSP is involved in centrosome separation and bipolar spindle assembly, whose silencing led to suppression of subcutaneous melanomas and ovarian tumors ${ }^{25}$. Simultaneous delivery of siRNAs targeting KSP and vascular endothelial growth factor (VEGF) has entered clinical trials, where the safety and efficacy of this combination is now being investigated ${ }^{26}$. The diverse nature of the chosen targets was intended to better extrapolate the significance of our outcomes. Pre-exposure to select siRNAs was undertaken using two different approaches. The "shock" treatment better mimics the clinical scenario where the malignant cells were exposed to a relatively high siRNA concentration immediately. This approach led to a selection of cells that were un-responsive to treatment for a variety of possible reasons: i) insufficient amount of intracellular siRNA due to low uptake; ii) insufficient silencing due to high level of mRNA and/or low RISC efficiency, which prevents reaching a critical level of silencing required for an effect on cell viability; and/or iii) reliance of cells on alternative proteins that minimizes the effect of silencing. The "gradual" siRNA treatment, on the other hand, affords the cell sufficient time to adopt and respond to siRNA treatments. Our previous studies indicated that siRNA effect on cell viability was complete after 72 hours $^{27}$, so that analysis at 24 hours was employed for the initial cellular response, while 72-hour analysis represented the response in surviving cells.

The RT-PCR analysis revealed interesting findings about the siRNA pre-treatment. First, the commercially obtained scrambled siRNA did affect the mRNA expression level of selected targets, especially KSP, at higher concentrations $(\geq 27 \mathrm{nM})$. The significant drop in cell viability observed with scrambled siRNA was in past attributed to toxicity of carriers, but it appears that this could be partially attributed to non-specific silencing by the scrambled siRNA. Secondly, the RT-PCR analysis performed after 24 hours during gradual siRNA exposure revealed significantly lower response of mRNA levels to repeated Mcl-1 and RPS6KA5 silencing for most of the exposure steps (but not for KSP). This decrease in responsiveness was not due to a higher target mRNA levels in pre-treated cells since the 72-hour analysis in the surviving cells clearly showed a lower mRNA level compared to the naïve cells. Therefore, we hypothesize that a lower response at the mRNA level might be due to temporary shortness of RISC 
complexes in cells pre-exposed to siRNA, which are immediately exposed to a repeat siRNA treatment. The higher mRNA levels of targeted proteins in surviving cells after the "shock" treatment (compared to the initial population; Figure 1c) might partially explain the survival of these cells (i.e., cells not displaying mRNA reduction might have survived better). However, a similar level of Mcl-1 and RPS6KA5 mRNA in the surviving and original cell population confirmed effective silencing of the target protein in surviving cells, which raises the possibility of the reliance of these cells on alternative survival pathways. The same similarity in mRNA levels, however, was not observed for KSP after 24 and 72 hours of treatment. The higher KSP mRNA levels in the surviving cells indicated the possibility of a lower silencing efficiency in this population, which could also led to better survival of the cells.

One obvious way for cells to adapt to siRNA treatment is by altering the uptake of siRNA complexes, reminiscent of resistance observed due to up-regulation of drug transporters. The siRNA uptake studies showed a similar trend in both "shock" and "gradual" siRNA treatments; even though some groups showed a lower level of siRNA internalization, the difference was relatively minor to rule out a significant change in cellular internalization of siRNA particles as a cause for any changes in siRNA response. The confocal imaging also confirmed similar internalization pattern and extent in selected cell populations. Later time point (72 hours) analysis demonstrated the expected release of internalized siRNA in a timely manner, which was not found to depend on siRNA pre-treatments. Confocal microscopy studies, however, indicated heterogeneity in the uptake of siRNA particles, where some cells had abundant complexes while others did not appear to contain any particles. It is likely that this variation might have contributed to survival of some cells after siRNA treatment. Flow cytometry analysis also indicated $<30 \%$ of cells with minimal (undetectable) siRNA, which confirms our microscopic observations.

A lower responsiveness in cell viability was observed after repeat siRNA treatment for the Mcl1/RPS6KA5 combination (shock and gradual treatment), but not to Mcl-1 and KSP siRNA treatment. However, the RT-PCR analysis showed a similar decrease in the mRNA levels of naïve and pre-treated 
cells in vitro. These findings indicate that the diminishing therapeutic response (i.e., loss of cell viability) to repeat treatment did not reflect a lowering silencing efficiency at the mRNA level. It is possible that overexpression or recruitment of an alternative mediator(s) for survival and proliferation of cells as a reaction to gradually increasing siRNA silencing. Selection of the cells that rely on alternative mediators as a result of shock siRNA treatment could be also a realistic possibility. Response in the animal model, however, was different and there was no indication of diminishing therapeutic response (either in the tumor growth pattern or target mRNA levels). We recognize the duration of these studies are relatively short (3 treatments) and longer-term studies might be required to unequivocally confirm this promising observation.

In order to investigate recruitment of alternative mediators, we analyzed the expression of a select group of proteins involved in cancer cell survival and proliferation. The selected proteins included another anti-apoptotic protein (survivin) ${ }^{28}$, JAK2 and STAT3 as the main effectors of JAK-STAT pathway $^{29}$, JUN, FOS, and MYC as downstream effectors of RAS-RAF pathway ${ }^{30}$, and NFkB, eIF4, and S6K as downstream effectors of PI3K-Akt pathway ${ }^{31,32}$. Interestingly, a significant upregulation in JUN and STAT3 levels, and to lesser extent in NFKB and JAK levels, was observed in survived cells after shock treatment with Mcl-1/RPS6KA5 siRNAs. It is noteworthy that the combination therapy seemed to have a more significant effect on the selected non-target proteins, which suggests a more likelihood for activating alternative pathways with silencing two targets simultaneously. The same trend was observed in gradually exposed cells (except for STAT3, where Mcl-1 silencing alone had a similar effect as the combinational silencing). The overexpression of non-target proteins in this case was observed after 24 hours, which is likely due to exposure to lower concentrations that were not sufficient for cell kill. Some reports indicated that cells could become more reliant on overexpressed pro-survival proteins in the face of drug threat ${ }^{33}$. We have also recently reported that cells overexpressing Mcl-1 as a result of resistance development to doxorubicin were more sensitive to Mcl-1 silencing ${ }^{34}$. Therefore, we investigated the silencing JUN, STAT3, NFKB, and JAK (as proteins overexpressed in the pre-treated cells) to evaluate 
the over-reliance on a specific mediator. The effect of siRNA silencing on cell viability was significantly enhanced when up-regulated JUN was targeted. Interestingly, this enhanced response was also more significant in cells pre-exposed to Mcl-1/RPS6KA5 siRNA combination compared to pre-exposure to Mcl-1 alone, which correlates with the RT-PCR analysis. The same trend was not observed with other over-expressed proteins. It seems that while the hypothesis of enhance response to silencing of overexpressed targets was confirmed in JUN overexpression, the conclusion cannot be generalized at this stage. Nevertheless, the high sensitivity to up-regulated JUN could provide another avenue for siRNA therapy if any resistance to therapy was seen in the xenograft model.

We are aware that the described studies might have certain limitations due to the need to maintain a reasonable scope for publication purposes. Our studies focused on a relatively few siRNA targets and utilized a particular type of non-viral polymeric carrier (lipid-modified PEI) for siRNA delivery. Our studies were conducted using a single cell line, MDA-MD-435 cells. Although the origin of this cell line has been questioned, ${ }^{35}$ the concept of resistance development affects different types of cancers and our results will be applicable to any cancer that will benefit from siRNA therapy. Additional studies with other siRNA targets, different types of non-viral carriers (e.g., lipids or polymers with different types of chemical modifications) and more cell lines will be needed to fully assess the impact of the reported observations.

In conclusion, the pre-treatment of cells with siRNA did not seem to significantly alter the extent of cellular internalization of siRNA-containing particles into target cells. While the decrease in mRNA levels of targeted proteins might not be as robust as the response in naïve cells as a result of repeated siRNA treatments (in particular in gradual treatments), the silencing efficiency was nevertheless restored if a 7-day recovery period was allowed for siRNA treated cells. The mRNA levels of targeted proteins also returned to original levels in a relatively short period of time (less than 2 weeks) in both "shock" and "gradual" treatments. Other mediators involved in breast cancer cell survival and proliferation (notably survivin, JUN, JAK2, NFkB and STAT3) were altered in siRNA treated cells, but only silencing 
JUN led to a heightened loss of viability. In vivo experiments in a xenograft model demonstrated a similar silencing efficiency at the mRNA level after each repeat dose, with no apparent resistance to the siRNA therapy. A longer-term investigation of repeated siRNA responses will be important to investigate to further explore limitations of siRNA therapy. 


\section{ACKNOWLEDGEMENTS}

The authors would like to thank Mrs. Geraldine Barron for her invaluable help with development and optimization of confocal microscopy, and Dr. Robert Clarke (Georgetown University, Washington, DC) for providing the MDA-435 cells. This project was financially supported by a Breast Cancer Research Grant from Canadian Breast Cancer Foundation (CBCF) and a NSERC Discovery Grant. 


\section{REFERENCES}

1. Boumendjel, A, Baubichon-Cortay, H, Trompier, D, Perrotton, T, Di Pietro, A (2005) Anticancer multidrug resistance mediated by MRP1: recent advances in the discovery of reversal agents. Med Res Rev 25:453-472.

2. Dean, M, Fojo, T, Bates, S (2005) Tumour stem cells and drug resistance. Nat Rev Cancer 5:275-284.

3. Gottesman, MM (2002) Mechanisms of cancer drug resistance. Annu Rev Med 53:615-627.

4. Bharadwaj, U, Marin-Muller, C, Li, M, Chen, C, Yao, Q (2011) Mesothelin confers pancreatic cancer cell resistance to TNF-alpha-induced apoptosis through Akt/PI3K/NF-kappaB activation and IL-6/Mcl-1 overexpression. Mol Cancer 10:106.

5. Buchholz, TA, Davis, DW, McConkey, DJ, Symmans, WF, Valero, V, Jhingran, A, et al. (2003) Chemotherapy-induced apoptosis and Bcl-2 levels correlate with breast cancer response to chemotherapy. Cancer J 9:33-41.

6. Sekine, I, Shimizu, C, Nishio, K, Saijo, N, Tamura, T, (2009) A literature review of molecular markers predictive of clinical response to cytotoxic chemotherapy in patients with breast cancer. Int J Clin Oncol 14:112-119.

7. Kanwar, RK, Cheung, CH, Chang, JY, Kanwar, JR (2010) Recent advances in anti-survivin treatments for cancer. Curr Med Chem 17:1509-1515.

8. Wagle, N, Emery, C, Berger, MF, Davis, MJ, Sawyer, A, Pochanard, P, et al. Dissecting therapeutic resistance to RAF inhibition in melanoma by tumor genomic profiling. J Clin Oncol 29:3085-3096.

9. Wykosky, J, Mukasa, A, Furnari, F, Cavenee, WK (2010) Escape from targeted inhibition: the dark side of kinase inhibitor therapy. Cell Cycle 9:1661-1662.

10. Soverini, S, Martinelli, G, Colarossi, S, Gnani, A, Rondoni, M, Castagnetti, F, et al. (2007) Second-line treatment with dasatinib in patients resistant to imatinib can select novel inhibitorspecific BCR-ABL mutants in Ph+ ALL. Lancet Oncol 8:273-274.

11. Romano, E, Pradervand, S, Paillusson, A, Weber, J, Harshman, K, Muehlethaler, K, et al. (2013) Identification of multiple mechanisms of resistance to vemurafenib in a patient with BRAFV600E-mutated cutaneous melanoma successfully rechallenged after progression. Clin Cancer Res 19:5749-5757.

12. Aliabadi HM, Landry B, Sun C, Tang T, Uludag H (2012) Supramolecular assemblies in functional siRNA delivery: where do we stand? Biomaterials 33:2546-2569.

13. Raemdonck, K, Vandenbroucke, RE, Demeester, J, Sanders, NN, De Smedt, SC (2008) Maintaining the silence: reflections on long-term RNAi. Drug Discov Today 13:917-931.

14. Mescalchin, A, Detzer, A, Wecke, M, Overhoff, M, Wünsche, W, Sczakiel, G.(2007) Cellular uptake and intracellular release are major obstacles to the therapeutic application of siRNA: novel options by phosphorothioate-stimulated delivery. Expert Opin Biol Ther 7:1531-1538.

15. Bartlett, DW, Davis, ME (2007) Physicochemical and biological characterization of targeted, nucleic acid-containing nanoparticles. Bioconjug Chem 18:456-468.

16. Zheng, ZM, Tang, S, Tao, M (2005) Development of resistance to RNAi in mammalian cells. Ann N Y Acad Sci 1058:105-118.

17. Aliabadi, HM, Maranchuk, R, Kucharski, C, Mahdipoor, P, Hugh, J, Uludağ, H. (2013)

Effective response of doxorubicin-sensitive and -resistant breast cancer cells to combinational siRNA therapy. J Control Release 172:219-228.

18. Incani, V, Tunis, E, Clements, BA, Olson, C, Kucharski, C, Lavasanifar, A, Uludag, H. (2007) Palmitic acid substitution on cationic polymers for effective delivery of plasmid DNA to bone marrow stromal cells. J Biomed Mater Res A 81:493-504. 
19. Neamnark A, Suwantong, O, Bahadur, RK, Hsu, CY, Supaphol, P, Uludağ, H. (2009) Aliphatic lipid substitution on $2 \mathrm{kDa}$ polyethylenimine improves plasmid delivery and transgene expression. Mol Pharm 6:1798-1815.

20. Aliabadi, HM, Landry, B, Bahadur, RK, Neamnark, A, Suwantong, O, Uludağ H. (2011) Impact of lipid substitution on assembly and delivery of siRNA by cationic polymers. Macromol Biosci 11:662-672.

21. Wright, CS (1984) Structural comparison of the two distinct sugar binding sites in wheat germ agglutinin isolectin II. J Mol Biol 178:91-104.

22. Booy, EP, Henson, ES, Gibson, SB (2011) Epidermal growth factor regulates Mcl-1 expression through the MAPK-Elk-1 signalling pathway contributing to cell survival in breast cancer. Oncogene 30:2367-2378.

23. Inuzuka, H, Fukushima, H, Shaik, S, Liu, P, Lau, AW, Wei, W. (2011) Mcl-1 ubiquitination and destruction. Oncotarget 2:239-244.

24. Anjum, R, Blenis, J (2008) The RSK family of kinases: emerging roles in cellular signalling. Nat Rev Mol Cell Biol 9:747-758.

25. Marra, E, Palombo, F, Ciliberto, G, Aurisicchio, L (2013) Kinesin spindle protein SiRNA slows tumor progression. J Cell Physiol 228:58-64.

26. Tabernero, J, Shapiro, GI, LoRusso, PM, Cervantes, A, Schwartz, GK, Weiss GJ, et al. (2013) First-in-humans trial of an RNA interference therapeutic targeting VEGF and KSP in cancer patients with liver involvement. Cancer Discov 3:406-417.

27. Aliabadi, HM, Landry, B, Mahdipoor, P, Hsu, CY, Uludag H (2012) Effective down-regulation of breast cancer resistance protein (BCRP) by siRNA delivery using lipid-substituted aliphatic polymers. Eur J Pharm Biopharm 81:33-42.

28. Altieri, DC (2008) Survivin, cancer networks and pathway-directed drug discovery. Nat Rev Cancer 8:61-70.

29. Klein, B, Tarte, K, Jourdan, M, Mathouk, K, Moreaux, J, Jourdan, E, et al. (2003) Survival and proliferation factors of normal and malignant plasma cells. Int J Hematol 78:106-113.

30. Chang F, Steelman, LS, Lee, JT, Shelton, JG, Navolanic, PM, Blalock, WL, et al. (2003) Signal transduction mediated by the Ras/Raf/MEK/ERK pathway from cytokine receptors to transcription factors: potential targeting for therapeutic intervention. Leukemia 17:1263-1293.

31. Bruno, B, Giaccone, L, Rotta, M, Anderson, K, Boccadoro, M (2005) Novel targeted drugs for the treatment of multiple myeloma: from bench to bedside. Leukemia 19:1729-1738.

32. Hernandez-Aya, LF, Gonzalez-Angulo, AM (2011) Targeting the phosphatidylinositol 3-kinase signaling pathway in breast cancer. Oncologist 16:404-414.

33. Yin Q, Shen, J, Chen, L, Zhang, Z, Gu, W, Li, Y. (2012) Overcoming multidrug resistance by co-delivery of Mdr-1 and survivin-targeting RNA with reduction-responsible cationic poly(beta-amino esters). Biomaterials 33:6495-6506.

34. Aliabadi, HM, Mahdipoor, P, Uludag, H (2013) Polymeric delivery of siRNA for dual silencing of Mcl-1 and P-glycoprotein and apoptosis induction in drug-resistant breast cancer cells. Cancer Gene Ther 20:169-177. 


\section{FIGURE LEGENDS}

Figure 1. Cell response to "shock" siRNA treatment and PCR analysis of targeted proteins. (a) Viability of MDA-435 cells (as a percentage of the "No Treatment" group) after 72 hours of exposure to siRNA complexes (54 nM). (b) Viability of naïve and "shock" siRNA treated cells after exposure to different siRNA concentrations. The designations on the x-axis indicate the siRNA used (C: Control siRNA, M: Mcl-1, M/R: Mcl-1/RPS6KA5 combination, and K: KSP) and its concentration in nM. Asterisks indicate significant difference compared to naïve cells. (c) The mRNA level of targeted proteins after 24 and 72 hours exposure to siRNA complexes $(54 \mathrm{nM})$. Asterisks indicate significant difference compared to 24 hours' time-point. The mRNA level of Mcl-1, RPS6KA5, and KSP in naïve and shock-treated cells after 7 days (d) and 14 days (e) of recovery. (f) The mRNA level of targeted proteins in "shock" siRNA treated cells (after 7 days of recovery) and naïve cells as the result of 24 hours exposure to siRNA complexes $(54 \mathrm{nM})$. In all cases, the levels of mRNAs in treated cells were normalized by the mRNA levels in nontreated (NT) cells.

Figure 2. Effect of "shock" siRNA pre-treatment on cellular internalization of siRNA complexes. The mean fluorescence (a) and percentage of FAM-siRNA positive cells (b) in treated cells compared to naïve non-treated cells. Asterisk indicates significant difference compared to control siRNA. (c) The confocal microscopy images after 24 and 72 hours exposure of siRNA complexes to (from left to right in both time-points) naïve cells, and cells pre-exposed to scrambled, Mcl-1, Mcl-1/RPS6KA5 combination, and KSP siRNAs. The scale bars represent $20 \mu \mathrm{m}$ in each picture. Staining for cell nucleus and membrane are indicated in blue and red, respectively.

Figure 3. Cell response to gradual siRNA treatments. (a) Viability of MDA-435 cells after 72 hours (as percentage of "No Treatment" group) of exposure to siRNA complexes. At $18 \mathrm{nM}$ siRNA treatment, the cells were already pre-treated with $9 \mathrm{nM}$ of siRNA targeting Mcl-1, Mcl-1/RPS6KA5 combination and KSP (except naïve cells). At 27 nM siRNA treatment, the cells were already pre-treated with 9 and 18 
nM of siRNA targeting Mcl-1, Mcl-1/RPS6KA5 combination and KSP (except naïve cells). (b) Viability of naïve and "gradual" siRNA treated cells after exposure to different siRNA concentrations. The "gradual" treated cells were allowed to recover for 7 days (from the last exposure to $27 \mathrm{nM}$ specific siRNA treatment) before the siRNA treatment. The designations on the $\mathrm{x}$-axis indicate the siRNA used (C: Control siRNA, M: Mcl-1, M/R: Mcl-1/RPS6KA5 combination, and K: KSP) and its concentration in nM. Asterisks indicate significant difference compared to naïve cells.

Figure 4. RT-PCR analysis of "gradual" siRNA treated cells for silencing efficiency. The mRNA levels of targeted proteins after 24 (left panels) and 72 hours (right panels) of siRNA treatment with gradually increasing concentrations $(9,18$ and $27 \mathrm{nM}$, followed by a repeat $27 \mathrm{nM}$ treatment), as indicated on individual figures. In all cases, the levels of mRNAs in siRNA treated cells were normalized by the mRNA levels in non-treated (NT) cells. Asterisks indicate significant difference compared to naïve cells.

Figure 5. RT-PCR analysis of cells recovered from "gradual" siRNA treatment for silencing efficiency. The mRNA levels of Mcl-1, RPS6KA5 and KSP in naïve and siRNA-treated cells after 7 (a) and 14 days (b) of recovery. Asterisks indicates significant difference compared to control siRNA. (c) The mRNA levels of targeted proteins in "gradual" siRNA treated cells ( 7 days of recovery) and naïve cells as the result of 24 hours exposure to siRNA complexes $(54 \mathrm{nM})$.

Figure 6. Effect of "gradual" siRNA pre-treatment on cellular internalization of siRNA complexes. The mean fluorescence (a) and percentage of FAM-siRNA positive cells (b) in treated cells compared to naïve non-treated cells. (c) The confocal microscopy images after 24 and 72 hours exposure of siRNA complexes to (from left to right in both time-points) naïve cells, and cells pre-exposed to scrambled, Mcl1, Mcl-1/RPS6KA5 combination, and KSP siRNAs. The scale bars represent $20 \mu \mathrm{m}$ in each picture. Staining for cell nucleus and membrane are indicated in blue and red, respectively. Asterisk indicates significant difference compared to control siRNA. 
Figure 7. A. RT-PCR analysis of non-targeted proteins in "shock" (a and c) and "gradual" (b and d) siRNA treated cells. The mRNA level of selected proteins were analyzed after 24 hours (a and b) and 72 hours (c and $\mathbf{d}$ ) of treatment with siRNAs against Mcl-1 and Mcl-1/RPS6KA5 combination. The mRNA levels of indicated proteins in siRNA treated cells were normalized against un-treated cells. Asterisks indicate a significant overexpression compared to no treatment. B. Cell viabilities of "shock" (a) and “gradual” (b) siRNA pre-treated cells after silencing for JAK, JUN, NFא-B, STAT3, MYC and S6K. The resultant cell viabilities were normalized with respect to the naïve cells. Red arrows indicate the significant increase in responsiveness of cells pre-exposed to Mcl-1 (SM and GM) or Mcl-1 and RPS6KA5 combination (SM/R and GM/R) to JUN silencing.

Figure 8. siRNA therapy in a xenograft model with a negative control siRNA and Mcl-1/RPS6KA5 siRNA combination. (a) Changes in tumor volumes treated with control siRNA and Mcl-1/RPS6KA5 siRNA combination. The siRNA injection was undertaken 3 times with 7 day intervals in between the injections (indicated as arrows). $\mathrm{n}=15,9$ and 3 for data points in week-1, week-2 and week-3, respectively. (b) RT-PCR analysis of tumor cells for the two targeted proteins (Mcl-1 and RPS6KA5) and the non-targeted JUN. The tumors were recovered either 2 or 7 days after siRNA injection for RTPCR analysis. The levels of the indicated mRNAs were normalized against the tumors treated with control siRNA. $\mathrm{n}=3$ at each data point. 